\title{
Excavation Balance Routing Algorithm Simulation Based on Fuzzy Ant Colony
}

\author{
Luo Xiaojuan, Yan Li \\ Pingxiang University, Pingxiang, Jiangxi, China, 337000
}

Keywords: Ant colony algorithm; Acfr routing algorithm; Exploration-excavation; Pheromone value

\begin{abstract}
This paper proposes a new fuzzy ant colony routing strategy based on ant colony algorithm to realize the exploration-excavation capability balance. It uses the fuzzy control system based on likelihood inference to calculate the local heuristic information. At the same time, according to the traditional ant colony routing algorithm, it calculates the pheromone value of different paths, and finally makes a routing decision based on the proportion of both.
\end{abstract}

\section{Introduction}

The DTN network describes an opportunity network that takes network segmentation and delay routing as normal [1-3]. Chapter 2 presents a FRA routing algorithm that has nothing to do with historical experience. The node obtains the metrics related to the current motion state, and obtains the current heuristic information value through the fuzzy inference system to help select the route. The premise of this method is that the network topology changes abnormally, and the historical experience of the node does not help the current situation $[4,5]$.

In fact, the initial changes in deployment are more serious. After being deployed to a suitable location, the topology structure will be relatively stable, thus forming a fully usable link for a period of time [6]. If you still blindly rely on local heuristic information, routing strategies that increase the transfer rate by increasing the number of copies will cause unnecessary communication costs. Therefore, we need to find an optimal balance strategy to meet the different situations where the topology structure is stable or changes drastically [7, 8].

Based on ant colony algorithm, this paper proposes a new type of Ant Colony based Fuzy Routing (ACFR) to achieve the balance of exploration and excavation capabilities. Heuristic information and Pheromone pheromone are the two most important concepts. The local heuristic information is calculated using a fuzzy control system based on likelihood inference; at the same time, the pheromone values of different paths are calculated according to the traditional ant colony routing algorithm. Finally, make a routing decision based on the proportion of the two.

\section{Acfr Routing Algorithm}

\subsection{Model construction}

The traditional routing algorithm based on ant colony modeling can't be applied to the air sensor network immediately. It can handle the exception of occasional link disconnection, but it can't adapt to the disconnected normal network. The ant colony algorithm focuses on the accumulation of historical experience, usually has a quick convergence, easy to stagnate and precocious, which makes it more adept at exploring the optimal solution, and is not conducive to exploring the entire solution space. DTN networks often require excellent exploration capabilities, using every contact opportunity around them to find possible forwarding opportunities. In the FRA, the heuristic information value is used as the only reference standard for the selection of forwarding nodes. It focuses on the current network conditions while ignoring the history of network routing experience.

ACFR uses a combination of the two methods. Path selection is determined not only by the pheromone value, but also by local heuristic information. The calculation of heuristic information is still determined by the fuzzy inference system, and the balance of the two uses a dynamic adaptive adjustment method. 
In ACFR, the node periodically sends a certain amount of ant agents to update the network pheromone table. Figure 1 shows this process. s represents the source node, and $d$ is the destination node. A certain amount of path finding ants go through the current node $i$ to find the path of the destination node. In the initial state, node i has a total of 4 neighbor nodes, and each has the same probability of selection. When the destination node $d$ is found, the pathfinding ant dies, generates a corresponding return ant, and returns from the original route according to the source route, and updates the pheromone value along the way. The ant sent from node $\mathrm{i}$ will release the pheromone pheromone on return.
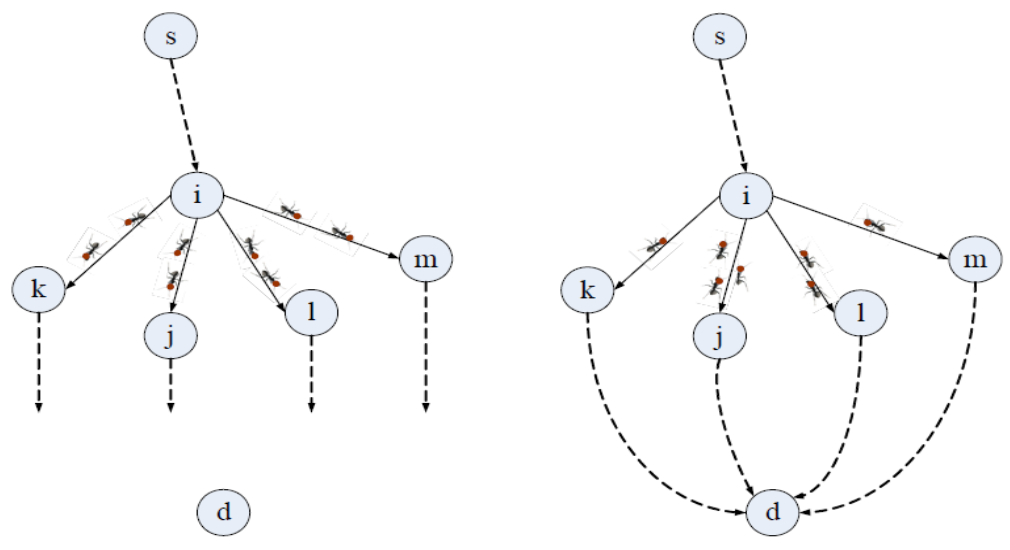

Fig. 1 Ant search path

Take node $\mathrm{i}$ as a reference point and examine edges (i, j). This indicates that there are a total of $n$ pheromone pheromone concentrations on the back $(i, j)$ of node $i$.

The routing algorithm degenerates into the FRA routing model. Under normal circumstances, it is necessary to weigh the proportion of the two and achieve adaptive adjustment.

\subsection{Adaptive exploration - digging the balance mechanism}

In order to obtain the minimum number of return ants, $\mathrm{n}_{\min }$, required to reach a certain probability value, first make the following assumptions:

(1) The first $n_{\min }$ return ants returning to node $i$ all come from edge (i, j). It makes sense to do this. If there are ants on the way from other neighbors, according to the previous formula, the concentration of pheromone pheromone in edge (i, j) becomes smaller, then the minimum number of ants to achieve a certain probability value must be greater than $\mathrm{n}_{\min }$.

(2) Calculate specific probability values using the traditional ant colony routing model without considering heuristic information values. The ACFR determines the value of the exploratory-excavation balance parameter based on the number of return ants. In the ideal case of communication, min $\mathrm{n}$ is the lower limit value of the required ant number. Considering heuristic information increases computational complexity, and the uncertainty of neighboring nodes makes it more difficult to evaluate.

\section{Simulation Experiment}

\subsection{Parameter setting}

In the experiment, each node cache is set to $250 \mathrm{M}$, the communication radius is $100 \mathrm{~m}$, and the SimpleBroadcast communication interface is used. The transmission speed is $250 \mathrm{~kb} / \mathrm{s}$. Nodes are at rest or in motion, with speeds ranging from 0 to $5 \mathrm{~m} / \mathrm{s}$. In addition, the message generator generates 1 packet every 10-15 intervals, and its size is 10 100k. Without loss of generality, the Random Waypoint motion model is used to randomly generate zig-zag paths that do not affect each other. The size of the simulation experiment area is $2000 \times 2000 \mathrm{~m}^{2}$.

Take $r=0.3$, set the probability threshold pupper and plower, respectively 0.7 and 0.3 , ACFR dynamically calculate nmin and n'min according to the number of neighbors. Taken $n_{f}=2 n_{\min }$, each time the network condition needs to be explored, the node sends nf path ants. When the return ant 
ratio $\varphi \geq 0.5$, the full ant colony routing model is used.

In addition to ACFR, the other five well-known routing algorithms implemented in ONE are also considered: First Contact (FC), Direct Delivery (DD), Epidemic (EP), Spray and Wait (SW), and Prophet (PR). For each strategy, multiple experiments were performed and then the statistical average results were compared.

\subsection{Analysis of results}

In the first set of experiments, a total of 90 nodes were placed in the area. Set the simulation time to 6000s. During this time, the message generator generated a total of 500 packets. Table 1 lists the total number of packets successfully delivered by each protocol. Since there is no limit on buffering and bandwidth, the EP passes 439 packets, greatly ahead of other routing tactics; ACFR passes a total of 416 packets, followed by it; while DD and FC, due to their simple implementation, only deliver separately. Because the total number of packets simulated by each protocol is the same, the more packets that are successfully delivered, the higher the success rate of delivery. Table 2 shows the statistical data of the success rate of the reporting module of the ONE simulator. It can be seen that this result is consistent with the trend of changes in Table 1.

Table 1 Comparison of Total Number of Successfully Passed Packets

\begin{tabular}{|c|c|c|c|c|c|c|}
\hline Time/Contrast & $1000 \mathrm{~s}$ & $2000 \mathrm{~s}$ & $3000 \mathrm{~s}$ & $4000 \mathrm{~s}$ & $5000 \mathrm{~s}$ & $6000 \mathrm{~s}$ \\
\hline Direct Delivery & 4 & 24 & 38 & 60 & 102 & 131 \\
\hline First Contact & 9 & 31 & 58 & 87 & 134 & 181 \\
\hline Epidemic & 38 & 125 & 206 & 281 & 357 & 339 \\
\hline Spray And Wait & 17 & 69 & 136 & 198 & 287 & 412 \\
\hline Prophet & 14 & 100 & 189 & 257 & 339 & 416 \\
\hline ACFR & 27 & 111 & 194 & 271 & 345 & 351 \\
\hline
\end{tabular}

Table 2 Comparison of Delivery Success Rate

\begin{tabular}{|c|c|c|c|c|c|c|}
\hline Time/Contrast & $1000 \mathrm{~s}$ & $2000 \mathrm{~s}$ & $3000 \mathrm{~s}$ & $4000 \mathrm{~s}$ & $5000 \mathrm{~s}$ & $6000 \mathrm{~s}$ \\
\hline Direct Delivery & $4.82 \%$ & $14.37 \%$ & $15.26 \%$ & $18.02 \%$ & $24.52 \%$ & $26.2 \%$ \\
\hline First Contact & $10.84 \%$ & $18.56 \%$ & $23.29 \%$ & $26.13 \%$ & $32.21 \%$ & $36.2 \%$ \\
\hline Epidemic & $45.78 \%$ & $74.85 \%$ & $82.73 \%$ & $84.38 \%$ & $85.82 \%$ & $87.8 \%$ \\
\hline Spray And Wait & $20.48 \%$ & $41.32 \%$ & $54.62 \%$ & $59.46 \%$ & $68.99 \%$ & $70.2 \%$ \\
\hline Prophet & $16.87 \%$ & $59.88 \%$ & $75.9 \%$ & $77.18 \%$ & $81.49 \%$ & $82.4 \%$ \\
\hline ACFR & $32.53 \%$ & $66.46 \%$ & $77.91 \%$ & $81.38 \%$ & $82.93 \%$ & $83.2 \%$ \\
\hline
\end{tabular}

In addition to transmission efficiency, the throughput cost of each strategy should also be compared, that is, the consumption of network resources. Here, the total number of data packets forwarded during the entire routing process is calculated, that is, the total number of data packet copies that are involved in forwarding when all links are available. The greater the number of copies, the greater the probability of delivery, but at the same time it will bring more unnecessary network consumption. As can be seen from Table 3, due to the use of a single-copy policy, DD only transfers data packets when it encounters the destination node, so its throughput is much smaller than other routing policies, and only a total of 131 packets are forwarded. Packets. The maximum transfer success rate reached by the EP is at the same time a great network throughput. A total of 39,082 data packets are transmitted in the 6000s, and this throughput is not allowed in practical situations. ACFR effectively achieves a balance between transfer rate and throughput, demonstrating superior performance. Another indicator of evaluation is the average delay of messages. As an important metric for evaluating the service quality of routing protocols, ACFR exhibits a satisfactory transmission speed compared with other routing protocols, as shown in Table 4. 
Table 3 Comparison of the Total Number of Forward Packets

\begin{tabular}{|c|c|c|c|c|c|c|}
\hline Time/Contrast & $1000 \mathrm{~s}$ & $2000 \mathrm{~s}$ & $3000 \mathrm{~s}$ & $4000 \mathrm{~s}$ & $5000 \mathrm{~s}$ & $6000 \mathrm{~s}$ \\
\hline Direct Delivery & 4 & 24 & 38 & 60 & 102 & 131 \\
\hline First Contact & 625 & 2222 & 4201 & 6305 & 8410 & 11089 \\
\hline Epidemic & 3399 & 11182 & 18251 & 25043 & 30959 & 39082 \\
\hline Spray And Wait & 344 & 851 & 1299 & 1723 & 2216 & 2707 \\
\hline Prophet & 519 & 4496 & 10729 & 17733 & 23659 & 30198 \\
\hline ACFR & 612 & 3903 & 7542 & 12352 & 17954 & 23583 \\
\hline
\end{tabular}

Table 4 Average Delay Comparison

\begin{tabular}{|c|c|c|c|c|c|c|}
\hline Time/Contrast & $1000 \mathrm{~s}$ & $2000 \mathrm{~s}$ & $3000 \mathrm{~s}$ & $4000 \mathrm{~s}$ & $5000 \mathrm{~s}$ & $6000 \mathrm{~s}$ \\
\hline Direct Delivery & 343.325 & 684.4458 & 798.4132 & 957.6117 & 1397.5412 & 1595.1992 \\
\hline First Contact & 203.6778 & 657.671 & 807.581 & 1008.8816 & 1140.6925 & 1480.9099 \\
\hline Epidemic & 339.4632 & 493.644 & 502.8621 & 501.8021 & 526.1983 & 548.3986 \\
\hline Spray And Wait & 292.6647 & 602.2246 & 798.9007 & 898.0364 & 1025.4631 & 1124.8764 \\
\hline Prophet & 315.9929 & 727.376 & 775.3735 & 753.0712 & 782.8947 & 808.9058 \\
\hline ACFR & 230.4123 & 520.1252 & 620.3421 & 631.6823 & 630.4183 & 632.324 \\
\hline
\end{tabular}

The second set of experiments tested the performance of routing protocols in different scale network environments. Keeping the simulation parameters unchanged, the message generator generated the same number of packets to be delivered within $6000 \mathrm{~s}$. The network scale grew from 20 nodes to 100 nodes. Compared with the entire simulation area, the coverage rate of one node is $0.79 \%$, and the network scale has increased from 15.8\% (20) to 79\% (100). Tables 5 and 6 respectively report the success ratios and average delays of routing protocols for different scales. In general, the greater the number of nodes, the higher the success rate of routing and the lower the average latency of packet delivery. This is because the more nodes there are, the more neighboring nodes there are and the greater the range of decision-making options. Regardless of the scale, ACFR has excellent performance. This is because ACFR mechanisms combined with pheromone values and heuristic information can be used to adapt to networks of different sizes: The fewer nodes are, the more sparse are the networks, and the more severe the network partition is. At this time, ACFR mainly relies on heuristic information for routing. The more nodes, the more dense the network, the more path selectivity, ACFR mainly rely on the historical experience embodied in pheromone to help the routing of data packets.

Table 5 Comparison of Delivery Success Rate

\begin{tabular}{|c|c|c|c|c|c|}
\hline Time/Contrast & 20 & 40 & 60 & 80 & 100 \\
\hline Direct Delivery & $27.24 \%$ & $27.89 \%$ & $28 \%$ & $26.92 \%$ & $28.66 \%$ \\
\hline First Contact & $29.42 \%$ & $25.9 \%$ & $31 \%$ & $34.01 \%$ & $32.87 \%$ \\
\hline Epidemic & $64.81 \%$ & $80.28 \%$ & $84 \%$ & $89.27 \%$ & $88.78 \%$ \\
\hline Spray And Wait & $55.47 \%$ & $63.15 \%$ & $69.6 \%$ & $73.08 \%$ & $71.74 \%$ \\
\hline Prophet & $50.7 \%$ & $72.51 \%$ & $79.6 \%$ & $83 \%$ & $85.57 \%$ \\
\hline ACFR & $60.02 \%$ & $78.1 \%$ & $82.57 \%$ & $84.96 \%$ & $87.41 \%$ \\
\hline
\end{tabular}

Table 6Average Delay Comparison

\begin{tabular}{|c|c|c|c|c|c|}
\hline Time/Contrast & 20 & 40 & 60 & 80 & 100 \\
\hline DirectDelivery & 1486.9781 & 1790.7386 & 1490.1836 & 1577.4654 & 1660.9699 \\
\hline FirstContact & 1765.1703 & 1780.3885 & 1578.6955 & 1621.6202 & 1496.8037 \\
\hline Epidemic & 1432.016 & 997.228 & 692.5231 & 596.5846 & 502.1158 \\
\hline SprayAndWait & 1501.1341 & 1316.0233 & 1141.4851 & 1122.5291 & 1106.6774 \\
\hline Prophet & 1815.1635 & 1493.5717 & 1002.0568 & 888.5717 & 744.9098 \\
\hline ACFR & 1500.432 & 1123.4243 & 843.1542 & 683.7091 & 596.2461 \\
\hline
\end{tabular}

In summary, the pheromone value used in ACFR is combined with heuristic information and adaptive weighting can be adapted to networks of all sizes. Experimental simulation proves its effectiveness.

\section{Conclusion}

Ant colony algorithm is a distributed self-organizing algorithm. Each ant's search process is 
independent of each other and communicates only through pheromones. It starts an independent solution search at multiple points in the problem space simultaneously, which not only increases the reliability of the algorithm, but also makes the algorithm have a strong global search capability.

This article discusses the ant colony routing algorithm. Compared with other strategies, the ant colony routing strategy does not require a high initial condition of the network. That is, the result of the ant colony algorithm does not depend on the selection of the initial route, and it does not require manual adjustment during the search process. It has strong robustness. Sex. Corresponding to the DTN network formed in the air, the local heuristic information valuesin the ant colony algorithm probability selection formulae will play an important role. The ACFR algorithm proposed in this chapter is an organic combination of FRA's fuzzy inference, and uses a dynamic exploration-excavation balance weight adaptive adjustment mechanism. Experimental simulations show that ACFR performs well in terms of transfer success rate and average delay compared to the traditional DTN routing protocol.

\section{References}

[1] Tan Ying. Calculate Group Intelligence Foundation [M]. Beijing: Tsinghua University Press. 2009:120-148.

[2] Dorigo, M., Maniezzo, V., Colorni. A. Ant system: optimization by a colony of cooperating agents [J]. IEEE Trans. Syst. Man Cybern. B, 1996, 26:29-41.

[3] Chen, G., Guo, T.D., Yang, W.G., Zhao, T. An improved ant-based routing protocol in Wireless Sensor Networks [C]. International Conference on Collaborative Computing: Networking, Applications and Worksharing, 2006:64.

[4] Kassabalidis, I., El-Sharkawi, M.A., Marks II, R.J., Arabshahi, P., Gray, A.A. Swarm intelligence for routing in communication networks [C]. GLOBECOM, 2001:3613-3617.

[5] Zhu, L., Li, Z., Cheng, Y., Xin, J. An Ants Intelligent Routing Protocol for Wireless Mesh Network [C]. International Conference on Wireless Communications, Networking and Mobile Computing, WiCom 2007: 1668- 1671.

[6] Kulkarni, R.V., Venayagamoorthy, G.K. Bio-inspired Algorithms for Autonomous Deployment and Localization of Sensor Nodes [J]. IEEE Trans. Syst. Man Cybern, 2010, 40:663-675.

[7] Kulkarni, R.V., Venayagamoorthy, G.K. Particle Swarm Optimization in Wireless-Sensor Networks: A Brief Survey [J]. IEEE Trans. Syst. Man Cybern. 2011, 41:262-267.

[8] Di Caro, G., Dorigo, M. Mobile agents for adaptive routing [C]. Proceedings of the 31st Hawaii International Conference on System Sciences, 1998, 7:74-84. 